\title{
Prácticas pedagógicas en el contexto de la cultura digital para generar aprendizajes significativos en los estudiantes 1
}

\author{
Jhenifer Mailec Rincón Guevara ${ }^{2}$ \\ Aurelia Patricia Quiñones Granados ${ }^{3}$
}

Recibido: 05-03-2017

Aceptado: 23-05-2017

\section{RESUMEN}

El presente artículo recoge los resultados de la investigación centrada en comprender las prácticas pedagógicas de los docentes de educación media en el contexto de la cultura digital, en dos instituciones oficiales de educación básica y media ubicadas en los departamentos del Cesar y Santander. En este trabajo se describe el proceso de implementación, el uso y apropiación de las tecnologías en la labor educativa, bajo una mirada fenomenológica de los sujetos participantes. Fue substancial conocer a profundidad qué sucede a diario en el quehacer pedagógico de los maestros cuando hacen uso de las tecnologías, sus aciertos y dificultades, con el fin de conocer qué tipo de formación y herramientas tecnológicas requieren, y qué apoyo se debe ofrecer para fortalecer este tipo de procesos que repercuten en la calidad educativa. Teniendo en cuenta los aportes teóricos de Olga Lucía Zuluaga, Pierre Lévy y David Ausubel.

Palabras clave: Prácticas pedagógicas, TIC en educación, cultura digital, aprendizaje significativo.

\footnotetext{
1. Artículo basado en el trabajo investigativo presentado para optar el título de magíster en Educación: Las prácticas pedagógicas en el contexto de la cultura digital para generar aprendizajes significativos en los estudiantes. Universidad Santo Tomás, 2014.

2. Licenciada en Lengua Castellana y comunicación. Magíster en Educación de la Universidad Santo Tomás de Bogotá. Tutora Programa Todos a Aprender MEN, docente catedrática Universidad Popular del Cesar.

Correo electrónico: jheniferrincon@gmail.com

3. Licenciada en Lengua Castellana y comunicación. Magíster en Educación de la Universidad Santo Tomás de Bogotá, 2014.

Correo electrónico: aureliaquinonez@hotmail.com
} 


\title{
Pedagogical practices in the context of digital culture to generate significant learning in students
}

\begin{abstract}
In this article presents the results of research based on understanding the pedagogical practices of teachers of secondary education in the context of digital culture, in two official institutions of primary and secondary education in the departments of Cesar and Santander. In this work the process described implementation, use and appropriation of technologies in educational work, under a phenomenological gaze of the subjects involved. It was substantial depth knowledge of what happens daily in the pedagogical work of teachers when they make use of technology, their successes and difficulties in order to know what kind of training and technological tools required, and what support should be offered to strengthen this type of processes that affect the quality of education.
\end{abstract}

Keywords: Pedagogical Practices, ICT in education, digital culture, meaningful learning.

\section{Introducción}

Comprender las prácticas pedagógicas de los docentes de educación media, en el contexto de cultura digital, y su relación con el fomento de aprendizajes significativos en los estudiantes; objetivo central de la investigación en mención, se desarrolló bajo una metodología con perspectiva fenomenológica, lo que nos permitió acercarnos a la realidad de las instituciones educativas estudiadas, a fin de entenderla y darle un significado. Para ello, se utilizó el método etnográfico (convencional y virtual), recurriendo a técnicas de recolección de datos como la observación participante y la entrevista.

Posteriormente, el análisis de los datos se realizó a partir de la triangulación de cada una de las categorías; práctica pedagógica, cultura 
digital y aprendizaje significativo, así como de la descripción de las subcategorías, del pensamiento de los autores, Zuluaga, Levy y Ausubel y de la interpretación propia de los investigadores.

Inicialmente, se aplicó una encuesta a los estudiantes a fin de hacer la caracterización inicial de la población e indicar con qué frecuencia hacían uso de ciertos dispositivos electrónicos; posteriormente, se llevó a cabo una entrevista a los docentes de las áreas de tecnología y matemáticas en las dos instituciones, se llenaron las rejillas de observación correspondientes a las clases de los mismos y se continuó con el seguimiento a las actividades en red de los docentes y estudiantes, por medio de la etnografía virtual.

Ahora bien, iniciamos dando respuesta al primer objetivo, el cual tuvo como fin identificar los tipos de prácticas pedagógicas de los docentes de educación media de las Instituciones Educativas Joaquín Ochoa Maestre y Gabriel García Márquez en el contexto de la cultura digital. Al respecto, cabe señalar que la descripción y el análisis de cada objetivo se realizará a partir de los datos proporcionados por los docentes en las entrevistas realizadas, quienes serán mencionados como $\left(D_{1}, D_{2}, D_{3}\right.$, $\mathrm{D}_{4}$ ), de los registros realizados en las rejillas de observación, las cuales se denominarán ( $R O D_{1}, R O D_{2}, R O D_{3}, R O D_{4}$ ) y de los pantallazos obtenidos de la etnografía virtual.

\section{Categoría: práctica pedagógica}

Según Zuluaga (1987), la práctica pedagógica se entiende como el campo de conocimiento en el que subyacen las formas de funcionamiento de los discursos en las instituciones educativas, y donde el maestro hace uso de todos los elementos propios de su formación académica y personal. Desde la academia, lo relacionado con el saber disciplinar y didáctico del docente, así como también con el pedagógico, es importante a la hora de reflexionar sobre las oportunidades y dificultades propias de su labor en el aula.

A fin de explicar y ampliar esta concepción, es necesario hacer un acercamiento a lo qué es el saber, la experiencia y el saber pedagógico, y a la forma cómo los abordaron los docentes que participaron en esta investigación. 


\section{Subcategoría: saber}

En cuanto a la subcategoría Saber, en el referente teórico planteado por Zuluaga (1987), el discurso asumido como "saber" tiene por finalidad metodológica el análisis de segmentos de discurso provenientes de diferentes prácticas, y el análisis de las mismas y de su existencia práctica en regiones del sabery del poder. De este modo, identificamos el saber que dominan los docentes partiendo de un pilar fundamental en su práctica, como es la formación docente, en la cual se ven enfocados a la educación formal, como se pudo evidenciar en la entrevista aplicada a los maestros participantes sobre su Saber en TIC:

D2: "La formación en TIC viene desde el pregrado en donde obtuve el título de Ingeniera de Sistemas, además, para reforzar mi práctica pedagógica he realizado una maestría relacionada con la Tecnología".

D3: "soy docente de tecnología e informática en la IE [...], vengo trabajando en esta parte desde el 2010 soy ingeniero electrónico de la UPC, también he recibido formación con el proyecto aula fundación aula telefónica, un aula especializada de tecnología en la cual me encuentro como coordinador de esta aula, además he recibido la capacitación de ciudadano digital estoy certificado como maestro digital y también estoy trabajando con la articulación con la media en el programa Técnico en Sistemas".

Los docentes alcanzan esta concepción de Saber en la medida de que desarrollan esta práctica en razón de su saber- poder, tal como se ejemplifica a continuación en los apartes tomados de las rejillas de observación:

RO. D3: el docente hace un repaso del tema de edición de imágenes y edición de video con programas como photoshop y movie maker. El docente hizo las aclaraciones pertinentes según las dudas que iban surgiendo de sus estudiantes, se percibe que el docente tiene apropiación conceptual de la temática expuesta, utiliza un lenguaje técnico propio del área de estudio.

Es muy notorio que en la subcategoría Saber los docentes dejan entrever un dominio conceptual de las temáticas que abordan en clase 
tanto en su discurso pedagógico como en la apropiación de diferentes estrategias y herramientas tecnológicas (programas, presentaciones) que han sido planeadas con un objetivo de enseñanza. Los docentes utilizan, entonces, un lenguaje técnico propio del área de estudio y se evidencia, además, un proceso de planeación acorde con los referentes de calidad, manejo de material y recurso tecnológico. Finalmente, los docentes proponen actividades que están organizadas a través de una secuencia de algoritmos debidamente explicados por el docente.

Teniendo en cuenta lo anterior, es importante resaltar el valor de la formación docente en la relación con el Saber, en cuanto a la integración de las TIC en las prácticas pedagógicas, puesto que implica un reto para el conocimiento educativo en la medida que dicha integración está fuertemente condicionada por los discursos que circulan en los ámbitos de la formación permanente del profesorado.

\title{
Subcategoría: experiencia
}

En relación con la subcategoría Experiencia, Zuluaga (1987) la define como el componente que soporta el método, como distintivo de su oficio, y su relación con el saber; es decir, consiste en enseñar por su relación con un saber y no por su relación con un método. En este contexto, "enseñar" es entendido como el oficio de los docentes, a quienes se les ha delegado la responsabilidad de aportar al desarrollo de las competencias cognitivas y sociales de las nuevas generaciones $y$, asimismo, de darle significado y sentido a la acción de enseñar en las relaciones que se tejen entre el maestro-alumno.

\begin{abstract}
D3: "Las TIC se integran de muchas maneras, por ejemplo, una de las oportunidades que he aprovechado son la gran acogida que han tenido las redes sociales en los estudiantes, en una ocasión creé unos grupos en Facebook en los cuales se crearon grupos de trabajo agregando a cada estudiantes y subí algunos videos de superación personal del área y les pedía que ellos me respondieran con comentarios y algunas preguntas para encausarlos a favor del aprendizaje y al desarrollo de competencias".
\end{abstract}

En cuanto a la experiencia, se observó que los docentes tienen una metodología de tipo instrumental; es decir, en el uso de las herramientas 
tecnológicas para dictar la clase, minimizan la función de los aparatos tecnológicos a la parte mediática donde se reemplaza el instrumento pero no hay trascendencia en la práctica. Al respecto, si bien los profesores utilizan los recursos tecnológicos de la institución, y hacen esfuerzos para llevar a cabo la implementación de las TIC, aún se requiere cambiar las prácticas docentes a fin de trascender la acción instrumental y magistral en la transmisión de conocimientos.

Por lo tanto, es un reto asumir la tecnología como una realidad gestada a través del sistema simbólico de la cultura en su conjunto, abandonando la concepción utilitarista de la tecnología como mero instrumento (González Quirós, 2004). En este sentido, es necesario, entonces, plantear unas nuevas prácticas innovadoras que apoyen aún más el proceso de los alumnos, en cuanto su autorregulación y capacidad de aprender por sí mismos.

\section{Subcategoría: saber pedagógico}

El saber pedagógico, según Zuluaga (1987) está conformado por los diferentes discursos a propósito de la enseñanza, entendida en la praxis educativa, y reúne los temas referentes a la educación, la instrucción, la pedagogía, la didáctica y la enseñanza.

D2: "Cuando se habla de aprendizaje [...] concluyo que es necesario construir conocimiento colectivo dentro de la práctica pedagógica, dada la naturaleza social del individuo".

D4 "Los procesos de aprendizaje requieren un poquito de todos los ingredientes que hasta hoy el hombre se ha preocupado por descubrir en temas relacionados con la pedagogía".

Los maestros que propician, en las escuelas, el espacio natural para la generación de saber pedagógico en el desarrollo de su profesión docente, toman decisiones sobre los procesos de su práctica pedagógica a partir de una reflexión crítica. Al respecto, los siguientes son algunos ejemplos extraídos de las rejillas de observación:

R.O D2: en varios momentos, la participación de los estudiantes es muy activa, propiciada por la estimulación del profesor a través de las preguntas que hace con el fin de 
recordar, elaborar, aplicar, analizar o evaluar conocimientos; hay una clara intencionalidad por parte del docente en hacer este tipo de pregunta tras pregunta con el fin de no permitir distracciones y mantenerlos concentrados en la temática, en ocasiones el profesor da pistas a las respuestas de las preguntas que el mismo hace, no deja ninguna pregunta suelta, cada pregunta que hace tiene un sentido para la construcción del conocimiento, el profesor no dicta definiciones, invita a los estudiantes que tomen apuntes sobre lo que les parezca más representativo de la clase, aunque en la guía de trabajo que dio a sus estudiantes, están los teoremas y definiciones del tema.

En esta subcategoría de saber pedagógico, se observó que el propósito de la enseñanza de las ciencias, utilizando múltiples estrategias, entre las que se encuentran aquellas que son prácticas y que se relacionan con recursos visuales e interactivos. Aun así, es necesario considerar los intereses, actitudes, habilidades y recursos que resulten motivantes para los estudiantes, a fin de que los procesos de aprendizaje sean significativos y potencien el desarrollo de las habilidades de los estudiantes.

Así lo afirma Zuluaga (1987), cuando menciona que el saber es el espacio más extenso y abierto de un conocimiento, donde convergen los diferentes discursos de los sujetos en la práctica. Ahora bien, este proceso implica el establecimiento de relaciones entre la realidad escolar y el entorno sociocultural que lo rodea, así como la aplicación y articulación de los saberes y herramientas que han sido dados al colectivo de docentes y que les permiten reflexionar sobre su quehacer en el aula con el fin de trascender en la práctica pedagógica. Para ello, entonces, es necesario partir de los saberes para los cuales han sido formados los docentes, de la constante actualización de conocimientos en relación con la experiencia y enseñanza, y de un proceso de autorreflexión de la labor docente en el aula.

\section{Categoría: cultura digital}

Es necesario caracterizar los componentes de la cultura digital en las prácticas pedagógicas, inicialmente, cabe destacar que entendemos por "cultura digital" el conjunto de sistemas culturales que han surgido del vínculo y la apropiación de las tecnologías, en los que la cultura no 
solo está determinada por las representaciones sociales que tienen los sujetos, sus formas de vida y sus valores culturales, sino que se tiene en cuenta los instrumentos y herramientas que la hacen posible; en este caso, los recursos tecnológicos que median la cultura digital.

A fin de abordar esta categoría, analizaremos los elementos que subyacen a La misma, entre los que se encuentran lo digital, lo virtual, la interactividad y la inteligencia colectiva.

\section{Subcategoría: digital}

Según Lévy (2007), lo que llamamos digital representa la información convertida en dígitos; es decir, cualquier imagen o secuencia de imágenes es traducible a una serie de dígitos.

En este caso, se observó el componente de la cultura digital que se materializa en la información que trabajan los jóvenes en la red, producto de la práctica pedagógica que desarrollan sus maestros.

\section{D1: "Sí, con software interactivo en construcción de gráficas de algunas funciones y diseño de circuitos eléctricos con datos reales de cada una de sus variables físicas (laboratorio virtual)".}

Se encontró que, a partir de las actividades que les sugieren a los estudiantes para desarrollar en la clase, los docentes utilizan los blogs y procesadores de datos, a los cuales deben ingresar la información a través de dígitos para que sea decodificada por el programa y muestre, por ejemplo las gráficas y los ejercicios.

\section{Subcategoria: virtual}

Lévy (2007), indica que lo virtual es aquello que existe en potencia pero no en acto y que tiende a actualizarse, aunque no se concretiza de un modo efectivo o formal. Es decir, "lo virtual no se opone a lo real sino a lo actual: virtualidad y actualidad sólo son dos maneras de ser diferentes. Para este autor, esto significa que lo virtual es real, pero que puede ser actual o no, puesto que es un proceso dinámico que se va construyendo día a día en el contexto digital y que, en algunos casos, cobra más fuerza que la realidad presencial. 
D3: "en el aula, utilizo redes sociales, correo electrónico, wiki, blogs, videos y objetos de aprendizaje, y la página oficial de la institución".

Siguiendo, esta subcategoría virtual, se observó que los docentes y estudiantes participan en un entorno virtual; es decir, los docentes proponen actividades en estos ambientes virtuales y los estudiantes participan con sus respectivas tareas en los blogs, en las redes sociales y en la plataforma institucional. Esto lo plantea Lévy (2007, p. 33) de la siguiente manera: "la transformación de una realidad en un conjunto de posibles"; es decir, la virtualidad sería otra forma de realidad que hace posible nuestra interacción con los otros.

\section{Subcategoría: interactividad}

En esta investigación, "interactividad" es definida como la participación activa del beneficiario de una transacción de información. El destinatario decodifica, interpreta, participa y moviliza su sistema nervioso de diversas maneras y siempre de manera diferente que su vecino (Lévy, 2007).

En relación con esta categoría, las evidencias otorgadas por los docentes entrevistados muestran que la interactividad a través de la Internet se da en un mayor porcentaje en la plataforma de la institución educativa y en los blogs que algunos docentes utilizan para su área (para la socialización de conocimientos y actividades), destacándose el papel moderador del docente en este tipo de actividades de interactividad.

D3: "Los estudiantes han tenido la oportunidad de participar en programas como el desarrollado por la Universidad Pontificia Bolivariana de Medellín para desarrollar competencias, denominado Divertic. Consistía en que los estudiantes hicieran producciones en videos donde mostraran actividades propias de la institución; en estas actividades, grabaron partidos de fútbol de los intercursos, hicieron videos con imágenes de actividades que se realizaban, como actos cívicos y culturales, y en el blog de tecnología en informática muestran las publicaciones de los trabajos hechos por los estudiantes, donde sus compañeros de curso realizan comentarios sobre los trabajos de sus compañeros; es una interactividad bien interesante donde las TIC son el medio ideal". 


\section{Subcategoría: inteligencia colectiva}

En este trabajo de investigación, se entiende por inteligencia colectiva a la inteligencia repartida en todas partes, valorizada constantemente, coordinada en tiempo real y que conduce a una movilización efectiva de las competencias. El objetivo de la inteligencia colectiva es el reconocimiento y el enriquecimiento mutuo de las personas, (Lévy, 2004).

Ahora bien, según los datos recogidos en las encuestas, se observa que, en cuanto a la inteligencia colectiva, los docentes y estudiantes sostienen una comunicación a través del entorno virtual, a partir de los vínculos sociales que les ha permitido establecer la construcción en forma colaborativa de videos, tutoriales, gráficas de funciones, presentaciones y más.

D-2: "Cuando se habla de aprendizaje, es necesario partir de la experiencia individual, la cual se vuelve colectiva al socializarse y se transforma en conocimiento al formalizarse durante la práctica pedagógica. Con lo anterior, concluyo que es necesario construir conocimiento colectivo dentro de la práctica pedagógica, dada la naturaleza social del individuo, quien aprende a trabajar colaborativamente con su par".

La inteligencia colectiva propende por la responsabilidad de cada uno de los miembros de la comunidad, porque no se trata de que cada individuo trabaje aisladamente y aporte, esporádicamente, ideas sueltas al conocimiento colectivo, sino que, por el contrario, se requiere del compromiso y de la claridad en el objetivo a alcanzar, ya que todos los miembros trabajan para un mismo fin, del cual todos se van a beneficiar. Es así como todos deben colaborar de forma transparentemente y con la claridad de saber qué se está haciendo, para qué y por qué. Los docentes manifestaron que, mediante el uso de herramientas tecnológicas y de software interactivo, se propician aún más los espacios para generar un conocimiento colectivo:

D-1: "En forma directa, cuando se utiliza el software interactivo en la construcción de gráficas y en el laboratorio virtual de los circuitos eléctricos, en los subgrupos, se fortalece el uso de los comandos propios del software y, a nivel grupal, la socialización de diferentes experiencias al trabajar el software y los alcances del mismo". 
Ahora bien, las tecnologías de la información y la comunicación (TIC) juegan un papel importante en el desarrollo de la inteligencia colectiva, puesto que en la red se encuentran espacios donde los miembros de una comunidad pueden dar sus aportes a fin de construir un conocimiento colectivo, como lo pudimos observar en los datos extraídos de la etnografía virtual realizada, en lo que se observa que los estudiantes se muestran participativos y sin prejuicios a la hora de dar un aporte que contribuya a la elaboración de conocimiento.

En cuanto al tercer objetivo, al establecer las relaciones entre las prácticas pedagógicas y el aprendizaje significativo, se encontraron los siguientes resultados:

\section{Categoría: aprendizaje significativo}

En el presente artículo, se entiende por "aprendizaje significativo" el producto de la relación de anclaje entre los pre-saberes y los conocimientos nuevos, generando una estructura cognoscitiva renovada y un aprendizaje significativo para la vida, ya que enriquecerá el conocimiento previo que se tenía. Al respecto, a continuación, se procederá a explicar cada una de las subcategorías en relación con los datos obtenidos a partir de las entrevistas a los docentes, las rejillas de observación (RO) y la etnografía virtual realizada.

\section{Subcategoría: estructura cognoscitiva}

En la presente investigación, la estructura cognoscitiva se entiende desde la teoría de Ausubel (1976), quien la concibe como el conjunto de conocimientos, ideas y conceptos que tiene el individuo sobre un saber específico y la organización de dichos conocimientos; así pues, la estructura cognoscitiva es una variable de suma importancia para el aprendizaje significativo, ya que si no se posee una estructura cognoscitiva clara y organizada no habrá significados precisos. Según este autor, la estructura cognoscitiva es una variable pertinente y decisiva que contribuye a facilitar el aprendizaje y a fortalecer los aspectos esenciales de la estructura cognoscitiva.

La estructura cognoscitiva es dinámica y se va modificando, organizando y reorganizando durante el proceso del aprendizaje 
significativo. Según Ausubel (1976), cada persona posee una estructura cognoscitiva única, con diferentes esquemas de organización, dependiendo de cómo se le facilite la comprensión del saber.

En la observación de las clases y en las entrevistas que se les realizó a los docentes, se observó que los estudiantes expresan ideas claras, elaboradas y coherentes con la temática que se aborda y que, además, participan activamente de las acciones sugeridas por el docente. De igual manera, el ambiente de la clase propicia confianza y seguridad en los dominios conceptuales.

Al respecto, en los datos recogidos en la rejilla de observación de clases se registró que:

RO D4: los estudiantes muestran tener conocimientos elaborados y acertados sobre el tema a tratar; la disposición de los estudiantes es adecuada; se muestran atentos, reflexivos, activos en la dinámica de preguntas y aportes a la clase.

Por otro lado, en las entrevistas realizadas, se pudo observar que los docentes no siempre toman como punto de partida la estructura cognoscitiva de los estudiantes para el proceso de aprendizaje, ya que si no ven relevante la información que el estudiante pueda tener simplemente la omiten. En este caso, se ven las dificultadas desde el inicio del proceso de aprendizaje, ya que si la información encontrada, no es la que el docente espera; de igual forma debe tenerse en cuenta, es en esta estructura cognoscitiva particular donde se va dar el anclaje de aprendizajes nuevos sobre una base de los ya existentes.

D-1: "hay estudiantes que, constantemente, están conectados a la red y consultan videos y artículos científicos relacionados con los contenidos de mi área; ellos me hablan sobre la información encontrada, la observo y si (veo que) es interesante y que aporta al conocimiento y que facilita el aprendizaje la socializamos al grupo".

Es importante que además del compromiso del docente exista por parte del estudiante una actitud y una disposición de su estructura cognoscitiva en el proceso de enseñanza- aprendizaje para que a su vez se construya el aprendizaje significativo. 
Al respecto, se registró en las rejillas de observación (RO) lo siguiente:

RO D1: "los estudiantes demuestran cierta desconcentración en el momento de observar el video, pero al momento de realizar los ejercicios en la pizarra digital su motivación cambia de tal forma que dicen las posibles respuestas, opinan sobre como resolverían el ejercicio y se disponen a realizarlo delante de sus compañeros".

\section{Subcategoría: subsunsores}

En la presente investigación, el concepto de "subsunsores" se entiende desde la teoría de Ausubel (1976), quien lo define como un conocimiento establecido en la estructura cognitiva del sujeto que aprende y que permite, por interacción, dar significado a otros conocimientos; es decir, los subsunsores son todos los saberes previos que posee el sujeto.

Las evidencias recolectadas en las observaciones de clase y en las entrevistas realizadas a los docentes muestran, en concordancia con lo anterior, la importancia del papel que ocupa el docente en la mediación cognitiva, al permitir el cambio de las estructuras mentales de los estudiantes y ofreciendo elementos fundamentales para el aprendizaje de tipo interno y externo.

RO D2: "los estudiantes muestran conocimientos previos acerca del tema trabajado (presentaciones Power Point), muchos de ellos participan activamente, hacen aportes como: "ya hay temas establecidos en PPT para las presentaciones, no hay necesidad profesora de hacerle cambios" "eso lo vimos el año pasado con la profesora..." "yo le hice a mi novia una presentación así con las fotos de los dos", estos conocimientos previos que muestran los estudiantes le sirven a la profesora para profundizar más sobre las opciones y funciones que tiene el programa, le da la oportunidad de enseñarles muchas otras cosas que ellos no sabían acerca del mismo como efectos, visualizaciones, inserción de temas musicales en las presentaciones, páginas web donde encuentran presentaciones PPT de cuentos, historias". 
En las entrevistas recogidas se evidenció que el establecimiento de los conocimientos previos de los alumnos como principio del proceso de aprendizaje no es otra cosa que activar sus experiencias anteriores con relación al tema nuevo que van a ver. En este sentido, los docentes participantes de esta investigación son conscientes de lo anterior y, por esto, gran parte del suministro para la planificación de sus clases consiste en esos saberes previos:

D-3 "Los estudiantes asocian los conocimientos previos con los nuevos que se les presentan, en la clase de edición de fotos y videos algunos tenían conocimiento que habían adquirido a través del manejo de su celular pero que fueron ampliados con las indicaciones dadas, ya que eran procesos más técnicos en la elaboración de dichos formatos de fotografía y video, montajes y aplicación efectos y se les dio la oportunidad que conocieran otros programas más completos sobre este proceso y también poder conocer cuáles eran compatibles o no con el sistema operativo de los equipos de cómputo".

De igual manera, se observó que los docentes aprovechan estos saberes previos de los estudiantes en el momento de sus prácticas docentes y buscan la forma de activarlos y de sacar el mayor provecho de ellos:

RO D4: "la participación de los estudiantes es muy activa; propiciada por la estimulación del profesor a través de las preguntas que hace con el fin de recordar, elaborar, aplicar, analizar o evaluar conocimientos."

\section{Subcategoría: transferencia}

En esta investigación, el proceso de transferencia es entendido como el anclaje de los nuevos conocimientos sobre los saberes previos, y la incorporación de la nueva información ya organizada con el conocimiento existente en la estructura cognoscitiva del estudiante, generando así una estructura cognoscitiva mejor organizada y renovada.

En relación con esta subcategoría de transferencia, se observan algunas consideraciones sobre la experiencia previa en relación con el nuevo aprendizaje en los estudiantes, las cuales favorecen este 
proceso mediante las TIC: al ofrecer estímulos de entrada a través de la presentación de contenidos en diferentes formatos que son decodificados por los estudiantes; mediante las redes de discusión y colaboración, considerando que el entorno social es fundamental para el aprendizaje (Vygotsky); al permitir personalizar los aprendizajes y el ritmo de los mismos, considerando las diferencias de los estudiantes.

\begin{abstract}
RO D1: "el ambiente de clase y las dinámicas de conversación y preguntas por parte del profesor fomentan una armonía apropiada para la adquisición de nuevos conocimientos, haciendo anclaje con los conocimientos que ya tenían los estudiantes y los conocimientos que obtuvieron del video visto; adicional a ello, las situaciones problema expuestas por el docente ayudaron a la organización de saberes y al aprendizaje de los estudiantes. Los estudiantes hicieron preguntas puntuales sobre lo observado en el video en el que no tuvieron mucha claridad, el docente procuró, en gran medida, hacer un recorrido paso a paso en el tablero sobre las fases de la resolución de las situaciones problema, haciendo uso de los teoremas y de las formulas vistas".
\end{abstract}

Sin embargo, se percibe que el discurso del docente cae, en algunos momentos, en prácticas tradicionalistas, con lo cual se tiende a perder la concentración en la clase por parte de los estudiantes. En este sentido, vemos que estas son situaciones que ameritan tenerse en consideración al momento de hacer la planeación de las clases, para que, de esta manera, sea el mismo docente quien se evalué en cada práctica pedagógica, ya que estos espacios de monotonía pueden cortar el proceso de aprendizaje significativo y una vez perdida la concentración del estudiante en este proceso de transferencia es muy difícil recuperarla.

RO D1: "el docente, en ocasiones, cae un discurso muy
tradicionalista; sin embargo, él mismo reacciona [cuando]
los estudiantes están perdiendo el hilo de las explicaciones y
retoma la dinámica de preguntas."

\title{
Subcategoría: significado
}

En esta investigación, se entiende el "significado" desde la teoría de Ausubel (1976), quien lo define como el producto del aprendizaje 
significativo. En esta subcategoría, los docentes y estudiantes dan sentido al producto de su proceso de aprendizaje significativo a través del empleo de las TIC, permitiendo que el estudiante sea: activo, constructivo, colaborativo, dialogado, contextualizado y reflexivo. En esta medida, el significado se concibe como el contenido cognoscitivo que tiene el estudiante después del proceso aprendizaje de un conocimiento.

En relación con el significado, las evidencias recogidas muestran cómo los docentes, en sus prácticas pedagógicas, tienen como objetivo principal explicar y dejarle claro a los estudiantes los aprendizajes que se derivan del conocimiento que están recibiendo; es decir, el proceso de mediación del docente es fundamental para que el estudiante considere y asimile el nuevo conocimiento a su estructura cognoscitiva, ajustándolo al que ya posee, y dándole importancia y relevancia desde diferentes perspectivas.

Recordemos que, desde la teoría del aprendizaje significativo, el docente es quien dirige el ritmo y el proceso del aprendizaje, es el mediador. El docente lleva a la práctica el saber transmitido en las clases, en este caso, aplicando teorías físicas en situaciones reales, dejando al estudiante explorar, construir y corroborar lo que en el aula se explicó, debatió y construyó.

No obstante, para que se produzca este significado, para que realmente exista un aprendizaje significativo, el alumno debe desarrollar, de manera autónoma, la capacidad de darle sentido a lo que aprende, demostrando, en sus producciones, lo que aprendió y complementó a sus conocimientos, gracias a las explicaciones del docente. El aprendizaje significativo es un proceso que se construye con la participación de varios elementos que entran a hacer parte de este gran proceso en igualdad de importancia, pues los conocimientos previos, la estructura cognoscitiva, la metodología utilizada por el docente mediador del aprendizaje, los conceptos, la interacción y el aprendizaje construido con los demás compañeros, el ritmo de aprendizaje de cada estudiante, la disposición del estudiante y el significado propio que el sujeto le dé a ese nuevo conocimiento es un todo que, en una misma mecánica de engranaje, va construyendo el aprendizaje significativo.

RO D1: "el docente hace proyecciones en su clase sobre conceptos teóricos que él mismo va leyendo; no hay una correcta utilización de las herramientas tecnológicas, 
desaprovecha los medios y cae en prácticas tradicionalistas nuevamente; aunque se esfuerza por hacer dinámica la clase, no hay una diferencia de tablero al video beam".

Se observa también que las clases se desarrollan en un ambiente de aprendizaje colaborativo, favoreciendo la comunicación y la relación entre los estudiantes y el profesor. En este sentido, si el docente, como mediador del aprendizaje, incentiva la participación activa de los estudiantes y se esfuerza por dar claridad a cada uno de los conceptos que menciona en su aula, los estudiantes perciben que su ambiente de trabajo es tranquilo y dinámico pese a la falta de recursos tecnológicos y de infraestructura.

\section{Propuesta sugerida}

El último objetivo de esta investigación es proponer estrategias para optimizar las prácticas pedagógicas en el contexto de la cultura digital. A continuación, se explicará en qué consiste la propuesta sugerida.

\section{Criterios pedagógicos}

Hasta el momento, hemos hecho un recorrido dentro de este proceso investigativo que incluye el análisis de los antecedentes y postulados teóricos de las categorías principales: práctica pedagógica, cultura digital y aprendizaje significativo, la aplicación de la metodología desde la perspectiva fenomenológica y el análisis de los resultados, a partir del cual, entonces, nace una propuesta pedagógica sugerida por los investigadores a fin de consolidar este proceso educativo y hacerlo más significativo para los estudiantes.

Al respecto, algunos criterios pedagógicos que presentamos para desarrollar una nueva práctica pedagógica, apoyada en las tecnologías digitales, que favorezca los aprendizajes, se centran en los elementos que han surgido a partir de la implementación de los objetivos de la investigación, los cuales buscan cambiar la práctica pedagógica tradicional por una práctica pedagógica colaborativa e interactiva, donde el estudiante no sea solo un usuario sino un productor de conocimientos. 
Se está aprendiendo un nuevo lenguaje, vivimos en una cultura digital, y es allí donde la escuela debe estar abierta a la nueva socialización del conocimiento, teniendo en cuenta experiencias significativas que le aporten al educando estrategias para avanzar en su construcción intelectual, emocional y humana; por ello, el compromiso debe ser el de asumir el reto como facilitadores del aprendizaje, encontrar la forma de seducir a los estudiantes y acercarlos a los contenidos de las clases.

Ahora bien, generar cambios en las prácticas pedagógicas implica tener conocimiento y exigirnos día a día estar a la vanguardia de lo que nos ofrece la tecnología, la innovación y las herramientas tecnológicas, por lo cual, proponemos que, desde el aula, se potencialice la interactividad y el trabajo colaborativo desde una ecología de medios y ambientes de aprendizaje adaptados a las necesidades circundantes de la escuela. Haremos una explicación breve de estos supuestos.

Interactividad: favorece la participación activa de los sujetos en una transacción de información donde el destinatario decodifica, interpreta, participa y moviliza su sistema nervioso de múltiples formas, siempre de manera diferente que su vecino, haciendo intercambio de saberes.

Ecología de medios: la ecología de medios o (media ecology) se presenta en el contexto de las teorías de la comunicación y busca comprender los cambios del sistema de medios. Postman (2000) utiliza la palabra "ecología" para indicar que no se centra solo en los medios, sino en las formas de interacción entre las personas; es decir, se parte del supuesto que con la tecnología se tiende a crear un nuevo ambiente de interacción entre los humanos.

El profesor Carlos Scolari, (2008) en su escrito Ecología de los medios. Mapa de un nicho teórico, explica cómo los ambientes estructuran lo que podemos ver y decir, y, por lo tanto, hacer. De acuerdo con este autor, los ambientes nos asignan roles e imponen la forma en la que debemos interpretarlos, especificando qué está permitido hacer y qué no lo está. En algunas ocasiones, por ejemplo, como en la corte, el aula o la oficina, las especificaciones son explícitas y formales, mientras que, en el caso de los medios que generan ambientes (por ejemplo, los libros, la radio, el cine, la televisión, etc.), las especificaciones son implícitas e informales y quedan semi-ocultas, porque asumimos que estamos en relación con una simple máquina y no con un ambiente (Postman, 1970). 
Al respecto, cabe destacar que la ecología de los medios busca explicitar estas especificaciones, permitiendo analizar cómo los medios de comunicación afectan nuestra percepción, comprensión, sensaciones y valores, y cómo nuestra interacción con los medios facilita una nueva forma de comunicación. Es así como la palabra "ecología" implica el estudio de ambientes: su estructura, contenido e impacto en la gente, ya que un ambiente es, después de todo, un sistema de mensajes complejos que impone a los humanos ciertas formas de pensar, sentir y comportarse (Postman, 1970).

Debemos ser conscientes, entonces, que los estudiantes que tenemos en nuestras aulas son estudiantes del siglo XXI: jóvenes y niños que serán los adultos del mañana y que nosotros como formadores, como responsables del compromiso social que tiene la escuela con la comunidad, debemos preparar a estos jóvenes para el futuro, enseñándoles a utilizar herramientas y contextos que hoy en día aún no existen. Es por esto que nuestra necesidad y preocupación por dar lo mejor de nosotros en las prácticas pedagógicas, y por utilizar los elementos necesarios para llegar a los jóvenes, debe ir más allá del deseo y la preocupación, ya que es urgente que transformemos nuestras habilidades y hagamos uso de lo que la red nos ofrece como herramientas didácticas de optimización de aprendizaje.

En este sentido, vale la pena meditar en el uso apropiado de las TIC para producir conocimientos, en las que las instituciones participantes se sientan beneficiadas de los aportes que se brinden mutuamente; herramientas como Educaplay, blogger y YouTube son sitios de la red, totalmente gratuitos, y si los utilizamos y los incorporamos a nuestras prácticas educativas proveerán un mejor ambiente de aprendizaje para los estudiantes, más dinámico y divertido. Debemos, entonces, repensar nuestras prácticas pedagógicas y contenidos curriculares a fin de lograr cambios sustanciales en los aprendizajes de nuestros estudiantes.

Para nuestro rediseño curricular nos basamos en las teorías del constructivismo, desde los diferentes puntos de vista de los exponentes de este modelo, las cuales, posteriormente, se aplicaron a la temática de la incorporación de las TIC en las prácticas pedagógicas, generando experiencias significativas en los estudiantes a partir de ambientes de aprendizajes colaborativos y cooperativos.

Ahora bien, nuestro diseño curricular se identifica con el concepto de currículo asumido por Coll (1987, p. 31): "es el proyecto que preside las 
actividades educativas escolares, precisa sus intenciones y proporción a guías de acción adecuadas y útiles para los profesores que tienen la responsabilidad directa de su ejecución".

Desde este marco, podemos iniciar explicando que la "significación" se entiende como la actividad mediante la cual es posible que el educando tome sus experiencias, y las transforme, con el fin de comunicarlas (específicamente relacionado con al área de lengua castellana); por lo tanto, desde esta perspectiva, la función esencial del lenguaje es la de establecer una comunicación con sentido, dando cuenta de una "conferencia discursiva"; de una competencia lingüística, la cual permite decodificar los enunciados según las reglas semánticas y gramaticales de la lengua; y de una competencia pragmática, que permite al lector o destinatario acceder a la interpretación del enunciado haciendo uso de las Tecnologías de la información y la comunicación.

En lo referente a la organización de los contenidos y al método para que el aprendizaje sea significativo, los estándares señalan la necesidad de reorganizar los contenidos por competencias y agrupar los temas de las diferentes competencias en unidades de sentido y ejes temáticos para que el aprendizaje sea relacionado y significativo, permitiéndole así al estudiante desarrollar niveles superiores en los procesos de pensamiento y establecer relaciones, diferencias, hipótesis, inferencias y síntesis a partir de los elementos que componen las redes conceptuales.

Por otra parte, se realizó una pequeña muestra de programación, la cual consistió en un replanteamiento de los temas y subtemas correspondientes a los cuatro ejes de estudio del grado sexto, enfatizando en los contenidos, sobretodo en el eje referido a los procesos culturales y estéticos asociados al lenguaje: el papel de la literatura.

Ahora bien, para la planeación y ejecución de la temática señalada se propusieron una serie de estrategias que permitan desarrollar dichos contenidos en función de los procesos y de la formación integral de los educandos. Para ello, es menester servir como mediador para incentivar el trabajo, de tal manera que sea el estudiante quien desarrolle por sí mismo procesos de tipo intelectual sicomotriz, afectivo y evolutivo, recurriendo al uso de las herramientas tecnológicas para que, de forma colaborativa, construya un aprendizaje significativo. 


\section{Conclusiones}

Las tecnologías de la información y de la comunicación han invadido el quehacer pedagógico desde hace algunos años, incorporando a las prácticas pedagógicas, nuevos y variados elementos que hacen una experiencia motivadora y enriquecedora. En esta medida, los docentes han experimentado cambios significativos en sus prácticas pedagógicas en los procesos de planeación, ejecución y evaluación de sus clases.

Entre los beneficios de la cultura digital emerge una gran cantidad de estrategias que buscan favorecer aprendizajes significativos en los estudiantes. La cultura digital es una realidad que está presente en la actualidad y que no va a desaparecer; por el contrario, a medida que pase el tiempo van a surgir muchas más herramientas y componentes $y$, por tanto, la educación no puede ser una isla aislada a esta realidad.

Como muestra de esto, los docentes partícipes de este trabajo de investigación, en sus prácticas pedagógicas, dejan ver lo deseosos que se encuentran de incorporar las herramientas digitales y las tecnologías de la información y la comunicación a sus clases, aprendiendo en el camino el uso pedagógico adecuado de las mismas, con el fin de construir un verdadero aprendizaje significativo en sus estudiantes. Ahora bien, gracias a esta investigación, se obtuvieron las siguientes conclusiones:

- Los estudiantes de hoy en día pasan la mayoría de su tiempo chateando, en video juegos online, hablando por celular, conociendo personas en la Internet y viendo TV, es decir, están inmersos en una cultura digital, de la cual el docente no saca el máximo provecho posible. En este sentido, los docentes no miden la potencialidad de estas habilidades que tienen los jóvenes y no saben aprovecharlas al momento de implementar sus prácticas pedagógicas.

- Las prácticas pedagógicas que incluyen las TIC, utilizadas adecuadamente en el contexto educativo, les da la oportunidad a los estudiantes de ser autónomos en las actividades que se sugieren trabajar. En este sentido, las herramientas digitales y software online favorecen la interactividad e inteligencia colectiva, ayudando a consolidar los saberes previos hasta convertirlos en significados debidamente elaborados. Sin 
embargo, pese al uso de las TIC, siguen predominando algunas prácticas de enseñanza basadas en modelos didácticos tradicionales no motivantes y significativas a los estudiantes.

- Los docentes, como usuarios de estas herramientas digitales y tecnológicas, tienen conocimientos básicos de su uso; sin embargo, su uso productivo, como herramienta en sus prácticas pedagógicas, varía de un maestro a otro, de acuerdo con su formación profesional, interés y actitudes.

Bajo esta perspectiva, emerge, cada vez con mayor contundencia, la necesidad de transformar las políticas de formación pedagógica para el uso de las TIC, a fin de incluir aspectos como una capacitación permanente y secuencial que cuente con horarios flexibles, acoplados a las particularidades del contexto, en donde prevalezcan contenidos útiles para la innovación didáctica, basados en la aplicación de estrategias relacionadas con el intercambio de experiencias docentes y el trabajo colaborativo en la red.

De igual manera, estas políticas deben recurrir al valioso recurso humano que se encuentra en los estudiantes, quienes son una poderosa fuerza de cooperación para llevar a cabo estos procesos de manera exitosa. Este es un reto urgente y primordial para el sistema educativo, dado que la generación actual está creciendo y desarrollándose en una sociedad en donde la tecnología es la que define el camino a seguir, pues los estudiantes que hoy en día tenemos en las aulas son los adultos del mañana, quienes seguramente se enfrentaran a empleos que hoy ni siquiera existen y por esto debemos prepararlos para el futuro con las herramientas que ellos disfrutan.

Es necesario pasar de lo instrumental a lo realmente significativo, es decir, desarrollar las prácticas docentes dentro del contexto de cultura digital. Hoy por hoy se hace necesario tomar conciencia de que los jóvenes nos llevan una gran ventaja en este aspecto, ya que ellos se desenvuelven en esta cultura, debido a que nacieron con una concepción del mundo de la que nosotros, como inmigrantes digitales, carecemos. En esta medida, la educación, como medio principal para el desarrollo integral de la sociedad al formar jóvenes ciudadanos competentes en este mundo de constantes cambios, debe estar al corriente de los adelantos de estas ciberculturas, así como los docentes deben ser responsables de vivir lo que viven sus estudiantes y de seguir siendo los orientadores de sus 
procesos de aprendizaje. Deben llegar a ellos partiendo de sus intereses, cambios, necesidades $y$, sobre todo, de lo que la sociedad necesita en el momento.

Por último, concluimos que este trabajo de investigación abrió muchas puertas y aclaró muchas dudas al interior de las comunidades educativas de las instituciones estudiadas, puesto que constituyó el primer paso para visualizar nuestras prácticas pedagógicas, evaluarnos y seguir mejorando a fin de brindar lo mejor a nuestros jóvenes. En este sentido, queda claro que, si bien estamos en el camino correcto, aún falta por construir en relación con las prácticas pedagógicas y la importancia que le brindamos a la cultura digital en la que hoy vivimos. Es por esto que la educación debe adaptarse a todos los cambios que estamos viviendo; debe enseñar a los estudiantes a pensar, aplicar, desarrollar, producir y analizar; para prepararlos para su futuro con las herramientas que hoy tenemos, que ellos disfrutan y que, además, optimizan la construcción de aprendizajes significativos, y aunque todo esto acarrea riesgos evidentes, el docente como mediador ejercerá estrategias prioritarias que los minimicen.

\section{Referencias bibliográficas}

Acevedo Zapata, S. (2014). Inclusión digital y educación inclusiva. Aportes para el diseño de proyectos pedagógicos con el uso de tecnologías de la comunicación. Revista De Investigaciones UNAD, 13(1), 41-57. doi:http://dx.doi.org/10.22490/25391887.1130

Acevedo Zapata, S. (2015). La formación de profesionales desde la reflexión en la acción con elementos fundamentales de la pedagogía crítica. Revista De Investigaciones UNAD, 14(1), 151 - 169. doi:http://dx.doi.org/10.22490/25391887.1350

Amaya Balaguera, Y. (2015). Guía metodológica ágil, para el desarrollo de aplicaciones móviles "AEGIS-MD". Revista De Investigaciones UNAD, 14(1), 97 - 113. doi:http:// dx.doi.org/10.22490/25391887.1348

Ausubel, D. P. (1976). Psicología Educativa, un punto de vista cognoscitivo. México: Editorial Trillas.

Burgos, J. (2011) Hacia la dinamización del currículo: necesidad de la escucha, la sospecha y la renovación. Disponible en: http://revistas.usta.edu.co/index.php/riiep/ article/view/1313

Congreso de la República. Ley 1341 (30, julio, 2009). Por la cual se definen Principios y conceptos sobre la sociedad de la información y la organización de las Tecnologías de la Información y las Comunicaciones -TIC-, se crea la Agencia Nacional del Espectro y se dictan otras disposiciones. Disponible en: http://www.mintic.gov.co/portal/604/w3article-3707.html 
Flores, A. (2014) Las tic en el aprendizaje universitario, según la edad. Disponible en: http://revistas.usta.edu.co/index.php/riiep/article/view/1326

Jiménez Mendoza, W., Luciano Alipio, R., \& Soto Carrión, C. (2014). Influencia de las fuentes de financiamiento externo en el crecimiento y desarrollo de las empresas agroindustriales del distrito de Abancay Apurímac Perú (periodo 2012- 2013). Revista De Investigaciones UNAD, 13(1), 261-273. doi:http://dx.doi. org/10.22490/25391887.1141

Lévy, P. (1998). ¿Qué es lo virtual? Barcelona: Editorial Paidós.

Lévy, P. (2004). Inteligencia colectiva. Por una antropología del ciberespacio. Disponible en: http://inteligenciacolectiva.bvsalud.org/public/documents/pdf/es/ inteligenciaColectiva.pdf

Lévy, P. (2007). Cibercultura. Informe al Consejo de Europa. Barcelona: Anthropos.

Moreira, M. A. (2010). ¿Al final, qué es aprendizaje significativo? (abril, 2010). Lección Inaugural del Programa de Posgrado en Enseñanza de las Ciencias Naturales. Instituto de Física de la Universidad Federal de Mato Grosso, Cuiabá. Disponible en: http:// moreira.if.ufrgs.br/alfinal.pdf

Régio, L., Egry, E., \& Apostólico, M. (2015). Consideraciones acerca de la importancia del estudio de saberes necesarios para el afrontamiento de la violencia infantil en la atención primaria de salud.. Revista De Investigaciones UNAD, 14(2), 137-149. doi:http://dx.doi.org/10.22490/25391887.1463

Sabogal Padilla, A. (2014). Retos de la pedagogía en el siglo XXI. Revista De Investigaciones UNAD, 13(2), 143-148. doi:http://dx.doi.org/10.22490/25391887.1154

Scolari, C. A. (2008). Hipermediaciones. Elementos para una teoría de la comunicación digital interactiva. Barcelona: Gedisa.

Zuluaga, O. L. (1987). Pedagogía e historia. Bogotá: Ediciones Foro Nacional por Colombia.

Zuluaga, O. L., Echeverri, A., Martínez, A., Restrepo, S., \& Quiceno, H. (1988). Pedagogía, didáctica y enseñanza. Educación y cultura, 14, 10-11. 